\title{
Treatments for Nail Psoriasis: A Systematic Review by the GRAPPA Nail Psoriasis Work Group
}

\author{
April W. Armstrong, William Tuong, Thorvardur J. Love, Sueli Carneiro, Rachel Grynszpan, \\ Steve S. Lee, and Arthur Kavanaugh
}

\begin{abstract}
Nail involvement in psoriatic diseases causes significant physical and functional disabilities. Evaluating, measuring, and treating nail involvement is important in improving the health outcomes and quality of life among patients with psoriasis and psoriatic arthritis (PsA). We performed a systematic analysis of the literature on nail psoriasis to help inform an update of treatment recommendations by the Group for Research and Assessment of Psoriasis and Psoriatic Arthritis (GRAPPA). (J Rheumatol 2014;41:2306-14; doi:10.3899/jrheum.140881)
\end{abstract}

Key Indexing Terms:

NAIL PSORIASIS TREATMENT THERAPY PSORIASIS EFFICACY EFFECTIVENESS

We performed 2 independent comprehensive literature searches of English-language human studies, published in the Medline database between January 1, 2006, and March 1, 2014, using the following search terms: psoriasis, psoriatic arthritis (PsA), nail, and treatment. Articles from the 2 searches were combined, and reference lists from articles from the database search were manually reviewed for additional relevant publications. Inclusion criteria were the following: adults (studies with $>5$ patients) with psoriasis or PsA and psoriatic nail involvement, and clinical trials, case series, or observational studies of therapies for psoriatic nail disease. Authors independently extracted the data, and any disagreements were adjudicated by consensus. Results are summarized below and presented fully in Tables 1A-1E.

From the Psoriasis Program, Department of Dermatology, Colorado Health Outcomes Program (COHO), University of Colorado Denver, Denver, Colorado; Department of Dermatology, University of California Davis, Davis, California, USA; Faculty of Medicine, University of Iceland, Department of Research, Landspitali University Hospital, Reykjavik, Iceland; Sector of Dermatology and Department of Medical Clinic, University Hospital and School of Medicine, Federal University of Rio de Janeiro, Rio de Janeiro, Brazil; Southern California Permanente Medical Group, Fontana, California; and University of California, San Diego, California, USA.

A.W. Armstrong, MD, MPH, Vice Chair for Clinical Research, Associate Professor of Dermatology, Director, Clinical Trials and Outcomes Research, Director, Psoriasis Program, Department of Dermatology, COHO, University of Colorado Denver; W. Tuong, BA, Department of Dermatology, University of California Davis; T.J. Love, $M D, P h D$, Faculty of Medicine, University of Iceland, Department of Research, Landspitali University Hospital; S. Carneiro, MD, PhD; R. Grynszpan, $M D$, Sector of Dermatology and Department of Medical Clinic, University Hospital and School of Medicine, Federal University of Rio de Janeiro; S.S. Lee, DO, FACR, Southern California Permanente Medical Group; A. Kavanaugh, MD, University of California.

Address correspondence to Dr. A.W. Armstrong, University of Colorado Denver, School of Medicine, Mail Stop 8127, 12801 East 17th Avenue, Aurora,CO 80045, USA.E-mail: aprilarmstrong@post.harvard.edu

\section{Topical Therapies $1,2,3,4,5$ (Table 1A)}

Topical therapies, an initial option for patients with mild nail involvement without significant functional impairment, include calcipotriol, a synthetic analog of vitamin D3 (50 $\mu \mathrm{g} / \mathrm{g}$ ), alone or in combination with betamethasone diproprionate. Limited evidence supports modest efficacy in psoriasis limited to $<2$ nails when used for $\geq 12$ weeks ${ }^{1,2,3}$. Moreover, twice daily calcipotriol monotherapy may have modest efficacy similar to daily calcipotriol and betamethasone diproprionate combination therapy.

Tacrolimus, a nonsteroidal topical calcineurin inhibitor that downregulates antigen-specific $\mathrm{T}$ cell activity and proinflammatory cytokine production, may have modest efficacy when applied once daily for $\geq 12$ weeks $^{4}$.

Tazarotene, a third-generation topical retinoid available as a cream or gel, may have a modest effect when used once daily in patients with nail bed and nail matrix lesions of moderate severity affecting $>2$ nails $\mathrm{s}^{1,5}$.

5-fluorouracil (5-FU), an antimetabolite that inhibits pyrimidine synthesis, has been used to treat actinic keratosis and squamous cell carcinoma in situ. However, topical 5-FU $1 \%$ lotion was no more effective than vehicle lotion when used daily for 12 weeks in patients with severe psoriatic nail dystrophy in $\geq 1$ nail $^{1}$.

\section{Procedural Therapies ${ }^{1,2,6,7}$ (Table 1B)}

The 595-nanometer pulsed dye laser (PDL) has been used to treat moderate-to-severe psoriatic nails monthly for $\geq 6$ months with limited efficacy ${ }^{6,7}$. Longer pulse durations (e.g., $6 \mathrm{~ms}$ vs $0.45 \mathrm{~ms}$ ) do not appear to result in greater efficacy and may cause greater side effects, such as pain ${ }^{6,7}$.

Limited evidence suggests that intralesional corticosteroid injections may be moderately effective in treating psoriatic nail dystrophies, particularly abnormalities of the nail matrix. However, studies vary on dosing and frequency, and many lack sufficient patient characteristics, e.g.,

Personal non-commercial use only. The Journal of Rheumatology Copyright @ 2014. All rights reserved. 
Table 1A. Topical therapies for nail psoriasis.

\begin{tabular}{|c|c|c|c|c|c|}
\hline Study & Therapy & $\begin{array}{l}\text { Study Type } \\
\text { and Population }\end{array}$ & Outcome Measure & $\begin{array}{l}\text { Patient Disease } \\
\text { Characteristics }\end{array}$ & Results \\
\hline $\begin{array}{l}\text { Rigopoulos } 2009^{2}, \\
\text { Greece }\end{array}$ & $\begin{array}{l}\text { Calcipotriol- } \\
\text { betamethasone } \\
\text { valerate ointment } \\
(\mathrm{qd} \times 12 \mathrm{wks})\end{array}$ & $\begin{array}{l}\text { Open-label, } \\
\text { uncontrolled; } \\
\mathrm{N}=25\end{array}$ & $\begin{array}{l}\text { NAPSI at } \\
\text { wks } 4,8,12\end{array}$ & $\begin{array}{l}\text { Mild cutaneous } \\
\text { psoriasis } \\
(\text { PASI }<10)\end{array}$ & $\begin{array}{l}\text { Mean NAPSI at baseline: } 5.8 \pm 1.7 \text {, } \\
\text { wk 12: } 1.6 \pm 0.6, \mathbf{p}<\mathbf{0 . 0 1} \mathbf{c} / \mathbf{w} \text { baseline }\end{array}$ \\
\hline $\begin{array}{l}\text { Tzung } 2008^{3} \text {, } \\
\text { Taiwan }\end{array}$ & $\begin{array}{l}0.005 \% \text { calcipotriol }+ \\
0.05 \% \text { betamethasone } \\
\text { diproprionate ointment } \\
(\mathrm{qd} \times 12 \mathrm{wks}) \text { vs } 0.005 \% \\
\text { calcipotriol ointment } \\
(\text { bid } \times 12 \mathrm{wks})\end{array}$ & $\begin{array}{l}\text { Randomized, } \\
\text { single-blinded, } \\
\text { comparator; } \\
\mathrm{N}=40\end{array}$ & $\begin{array}{l}\text { Investigator Global } \\
\text { Assessment Score } \\
\text { (IGA), NAPSI at } \\
\text { wks } 0,4,8,12\end{array}$ & $\begin{array}{l}\text { Fingernail psoriasis } \\
\text { (severity not } \\
\text { mentioned) }\end{array}$ & $\begin{array}{l}\text { IGA after } 12 \text { wks ( } \% \text { patients with } \geq \\
\text { moderate improvement): calcipotriol }+ \\
\text { betamethasone: } 53 \% \text {, calcipotriol: } 53 \%, \\
\mathbf{p}=\mathbf{0 . 0 7 1} \text { btwn groups; mean NAPSI after } 12 \text { wks: } \\
\text { specific values NR; } \mathbf{p}=\mathbf{0 . 6 4 9} \text { btwn groups }\end{array}$ \\
\hline $\begin{array}{l}\text { De Simone } 2013^{4} \text {, } \\
\text { Italy }\end{array}$ & $\begin{array}{l}0.1 \% \text { tacrolimus ointment } \\
(\mathrm{qd} \times 12 \mathrm{wks}) \mathrm{vs} \\
\text { no treatment }\end{array}$ & $\begin{array}{l}\text { Randomized, } \\
\text { controlled, } \\
\text { open-label; } \\
\mathrm{N}=21\end{array}$ & $\begin{array}{l}\text { NAPSI at wks } \\
0,6,12\end{array}$ & $\begin{array}{l}\text { Fingernail psoriasis } \\
\text { (severity not } \\
\text { mentioned) }\end{array}$ & $\begin{array}{l}\text { Mean NAPSI at baseline vs wk } 12: 0.1 \% \\
\text { tacrolimus: } 23.0 \text { vs } 10.0 \text {, No treatment: } 19.3 \text { vs } \\
16.3, \mathbf{p}<\mathbf{0 . 0 0 1} \text { btwn groups }\end{array}$ \\
\hline
\end{tabular}

Data in bold face are p values.bid: twice daily; btwn: between; c/w: compared with; bl: baseline; qd: every day; N: number; NAPSI: Nail Psoriasis Severity Index; PASI: Psoriasis Area and Severity Index; NR: not reported.

Table 1B. Procedural therapies for nail psoriasis.

\begin{tabular}{|c|c|c|c|c|c|}
\hline Study & Therapy & $\begin{array}{l}\text { Study Type } \\
\text { and Population }\end{array}$ & Outcome Measure & Baseline & Results \\
\hline $\begin{array}{l}\text { Goldust } 2013^{6} \text {, } \\
\text { Iran }\end{array}$ & $\begin{array}{l}\text { Pulsed dye laser }(595 \mathrm{~nm} \text {, } \\
7 \text {-mm spot size, } 0.45 \mathrm{~ms} \text { pulse } \\
\text { duration, } 6 \mathrm{j} / \mathrm{cm}^{2}, 20 \mathrm{~ms} \text { cryogen } \\
\text { spurt with } 10 \mathrm{~ms} \text { delay, qm } \\
\times 6 \mathrm{mo} \text { ) vs same, except } 6 \mathrm{~ms} \\
\text { pulse duration, } 9 \mathrm{j} / \mathrm{cm}^{2}\end{array}$ & $\begin{array}{l}\text { Randomized, } \\
\text { double-blinded, } \\
\text { intrapatient, } \\
\text { left-to-right; } \mathrm{N}=40\end{array}$ & $\begin{array}{l}\text { NAPSI at mo } \\
0,1,2,3,4,5,6\end{array}$ & $\begin{array}{l}\text { Mild-to-moderate plaque } \\
\text { psoriasis and refractory } \\
\text { nail involvement, } \leq 30 \% \\
\text { BSA of plaque psoriasis, } \\
\text { no active PsA or pustular } \\
\text { psoriasis of nail }\end{array}$ & $\begin{array}{l}\text { Significant decrease in mean } \\
\text { NAPSI, nail matrix NAPSI, } \\
\text { and nail bed NAPSI at } 6 \text { mo } \\
\text { c/w baseline in both groups; } \\
\text { specific values NR. NS btwn } \\
\text { groups }\end{array}$ \\
\hline
\end{tabular}

Data in bold face are p values; btwn: between; c/w: compared with; qm: every month; mo: month(s); BSA: body surface area; NAPSI: Nail Psoriasis Severity Index; NR: not reported; NS: not significant; PASI: Psoriasis Area and Severity Index.

severity and type of psoriatic disease ${ }^{1}$. Typically, 0.05-0.3 $\mathrm{ml}$ of triamcinolone acetonide $2.5-10 \mathrm{mg} / \mathrm{ml}$ is injected at multiple sites in the proximal nailfold at weekly intervals for $\leq 5$ months $^{2}$.

\section{Traditional Oral Systemic Therapies ${ }^{1,8-16}$ (Table 1C)}

Although traditional systemic therapies have not been rigorously tested, oral cyclosporine, an immunosuppressant drug that interferes with activity and growth of $\mathrm{T}$ cells, has modest efficacy in nail psoriasis ${ }^{1,8,9,10,11}$. Oral methotrexate (MTX, $\leq 15 \mathrm{mg}$ weekly), an antimetabolite and antifolate drug commonly used to treat psoriasis and inflammatory arthritis, has been tested rigorously, but is unlikely to result in significant improvement in psoriatic nail disease $8,9,12,13,14$. Briakinumab [an interleukin 12/23 (IL-12/23) inhibitor no longer in development] was superior to MTX in 1 study $^{13}$. Acitretin, a second-generation retinoid and a metabolite of etretinate, had modest efficacy at doses of $0.2-0.3$ $\mathrm{mg} / \mathrm{kg} /$ day for 6 months $\mathrm{s}^{1,9,14,15}$. Leflunomide, an oral pyrimidine synthesis inhibitor, also had modest efficacy in psoriatic nail dystrophy when dosed at $100 \mathrm{mg} /$ day for 3 days, then $20 \mathrm{mg} /$ day for 24 weeks ${ }^{16}$.

\section{Biologic Therapies ${ }^{1,9,11,14,17-41}$ (Table 1D)}

Tumor necrosis factor- $\alpha$ (TNF- $\alpha$ ) plays a key role in the pathogenesis of psoriasis and PsA, and can interrupt TNF signaling, thereby leading to improvements in nail dystrophy. In several controlled studies, adalimumab $(\mathrm{ADM})^{9,11,14,17,18,19,20,21,22,23,24}$, certolizumab pegol $^{25}$, etanercept ${ }^{9,14,22,23,24,26,27}$, golimumab ${ }^{28,29}$, and inflixi- 
Table 1C. Traditional oral systemic therapies for nail psoriasis.

\begin{tabular}{|c|c|c|c|c|c|}
\hline Study & Therapy & $\begin{array}{l}\text { Study Type } \\
\text { and Population }\end{array}$ & Outcome Measure & Baseline & Results \\
\hline $\begin{array}{l}\text { Gumusel } 2011^{8} \text {, } \\
\text { Turkey }\end{array}$ & $\begin{array}{l}\text { CsA (initial } 5-\mathrm{mg} / \mathrm{kg} \\
\text { dose PO qd } \times 12 \text { wks } \rightarrow \\
2.5-3.5 \mathrm{mg} / \mathrm{kg} \text { PO qd } \times 12 \\
\text { wks) vs MTX (initial } 15-\mathrm{mg} \\
\text { dose SQ qw } \times 12 \text { wks } \rightarrow 10 \mathrm{mg} \\
\text { SQ qw } \times 12 \text { wks) }\end{array}$ & $\begin{array}{l}\text { Randomized, } \\
\text { single-blinded, } \\
\text { comparator, } \mathrm{N}=37\end{array}$ & $\begin{array}{l}\text { NAPSI at wks } \\
0,4,8,12,16,20, \\
\text { and } 24\end{array}$ & $\begin{array}{l}\text { Psoriatic patients with } \\
\text { nail involvement, } \geq 10 \% \\
\text { of BSA with lesions, } \\
\text { PASI } \geq 10 \text {, NAPSI } 10 \\
\text { or psoriatic patients with } \\
\text { nail involvement distressed } \\
\text { because of either their condition } \\
\text { or their nail pathology that } \\
\text { proved to be resistant to } \\
\text { topical treatment regardless of } \\
\text { BSA and PASI }\end{array}$ & $\begin{array}{l}\text { Mean NAPSI score at wk 0: CsA: } \\
42.1 \pm 26.4, \text { MTX: } 39.1 \pm 19.9 \\
\text { wk 24: CsA: } 25.4, \text { MTX: } 18.3 \\
\text { NS btwn groups }\end{array}$ \\
\hline $\begin{array}{l}\text { Sanchez-Regana } \\
2011^{9} \text {, Spain }\end{array}$ & $\begin{array}{l}\text { Classical treatments } \\
\text { [acitretin (PO), MTX } \\
\text { (PO or SQ) CsA (PO), } \\
\text { PUVA, NB-UVB, } \\
\text { Re-PUVA, Re-NB-UVB] vs } \\
\text { biological treatments } \\
\text { (IFX; IV), (ETN; SQ), } \\
\text { efalizumab (SQ), or (ADM; SQ) }\end{array}$ & $\begin{array}{l}\text { Retrospective review, } \\
\mathrm{N}=84\end{array}$ & $\begin{array}{l}\text { NAPSI at weeks } \\
12,24 \text {, and } 48\end{array}$ & $\begin{array}{l}\text { Moderate-to-severe } \\
\text { psoriasis (PASI } \geq 3 \text { ), } \\
\text { PsA, and presence of } \\
\text { psoriasis of the nails }\end{array}$ & $\begin{array}{l}\text { Mean percent change in } \\
\text { NAPSI score: wk 48: Classical: } \\
\text { CsA: } 89.1 \% \text { (p value vs CsA), } \\
\text { Acitretin: } 51.7 \%, \mathbf{p}<\mathbf{0 . 0 0 1 ,} \\
\text { MTX: } 34.9 \%, \mathbf{p}<\mathbf{0 . 0 0 1 ,} \\
\text { PUVA: } 69.1 \%, \mathbf{p}=\mathbf{0 . 0 2 3}, \\
\text { NB-UVB: } 5.0 \%, \mathbf{p}<\mathbf{0 . 0 0 1 ,} \\
\text { Re-PUVA: } 84.6 \%, \mathbf{p}=\mathbf{0 . 1 9 0 ,} \\
\text { Re-NUVB: } 64.4 \%, \mathbf{p}=\mathbf{0 . 0 0 3 ,} \\
\text { Biological: IFX: } 91.5 \% \\
\text { (p-value vs IFX), ETN: } 86.7 \%, \\
\mathbf{p}=\mathbf{0 . 4 2 3} \text {, Efalizumab: } 82.5 \%, \\
\mathbf{p}=\mathbf{0 . 2 3 7} \text {, ADM: } 84.2 \%, \\
\mathbf{p}=\mathbf{0 . 0 8 3 , ~ M e a n ~ p e r c e n t ~ c h a n g e ~ i n ~} \\
\text { NAPSI score at wk } 48: \text { Classical: } \\
57.2 \%, \text { Biological: } 86.0 \%, \\
\mathbf{p}<\mathbf{0 . 0 0 1} \text { btwn groups }\end{array}$ \\
\hline Syuto $2007^{10}$, Japan & $\begin{array}{l}\text { CsA (initial 3-mg/kg dose PO } \\
\text { bid } \rightarrow 1.5 \mathrm{mg} / \mathrm{kg} \text { PO qd if } \\
\text { improvement) }\end{array}$ & $\begin{array}{l}\text { Open-label, } \\
\text { uncontrolled, } \mathrm{N}=16\end{array}$ & Improvement & $\begin{array}{l}\text { Duration of psoriatic } \\
\text { nails ranged from } 1-27 \text { years. } \\
13 / 16 \text { patients were } \\
\text { unresponsive to prior } \\
\text { treatments }\end{array}$ & $\begin{array}{l}\text { 2/16 complete resolution; } 10 / 16 \\
\text { significant improvement; } 3 / 16 \\
\text { slight improvement; } 1 / 16 \text { no } \\
\text { change }\end{array}$ \\
\hline $\begin{array}{l}\text { Reich } 2011^{13} \text {, } \\
\text { Germany, Canada, } \\
\text { France }\end{array}$ & $\begin{array}{l}\text { MTX (5-25 mg PO qw } \\
\text { x52 wks) vs Briakinumab } \\
\text { (initial 200-mg dose SQ at } \\
\text { wks } 0 \text { and } 4 \rightarrow 100 \text { mg SQ } \\
\text { q } 4 \text { wks, wks 8-48) }\end{array}$ & $\begin{array}{l}\text { Randomized, } \\
\text { double-blinded, } \\
\text { comparator, } \mathrm{N}=317\end{array}$ & $\begin{array}{l}\text { NAPSI (target } \\
\text { fingernail) at wks } \\
0,24,52\end{array}$ & $\begin{array}{l}\text { Psoriasis for } \geq 6 \text { months } \\
\text { and stable plaque psoriasis } \\
\geq 2 \text { months, PGA } \geq 3 \text {, } \\
\text { PASI } \geq 12,10 \% \text { BSA } \\
\text { affected by psoriasis, }\end{array}$ & $\begin{array}{l}\text { Mean NAPSI score (target } \\
\text { fingernail) at wk 0: MTX: } 4.8 \pm \\
\text { 2.1, Briakinumab: } 4.8 \pm 2.0 \text {; } \\
\text { wk 52: MTX: } 3.0 \text {, Briakinumab: } \\
\text { 1.2, p < } \mathbf{0 . 0 0 1} \text { btwn groups }\end{array}$ \\
\hline Tosti $2009^{15}$, Italy & $\begin{array}{l}\text { Acitretin }(0.2-0.3 \mathrm{mg} / \mathrm{kg} \\
\text { PO qd ×6 months })\end{array}$ & $\begin{array}{l}\text { Open-label, } \\
\text { uncontrolled, } \mathrm{N}=36\end{array}$ & $\begin{array}{l}\text { NAPSI at } \\
\text { months } 0,2,4,6\end{array}$ & $\begin{array}{l}\text { Moderate-to-severe psoriasis } \\
\text { limited to the nails }\end{array}$ & $\begin{array}{l}\text { Mean NAPSI score at baseline vs } \\
\text { month 6: } 31.5 \text { (range 10-46) vs } \\
\text { 18.6 (range 6-34); percent } \\
\text { reduction of NAPSI score at } \\
\text { month 6: } 41 \%\end{array}$ \\
\hline $\begin{array}{l}\text { Behrens } 2013^{16}, \\
\text { Germany, Czech } \\
\text { Republic, Slovenia }\end{array}$ & $\begin{array}{l}\text { Leflunomide (initial } 100-\mathrm{mg} \\
\text { dose PO qd } \times 3 \text { days } \rightarrow 20 \mathrm{mg} \\
\text { PO qd } \times 24 \text { wks) }\end{array}$ & $\begin{array}{l}\text { Observational, } \\
\mathrm{N}=514\end{array}$ & $\begin{array}{l}\text { Clinical severity } \\
\text { (5-pt scale) }\end{array}$ & $\begin{array}{l}\text { Active psoriatic disease; } \\
\text { no previous leflunomide } \\
\text { treatment }\end{array}$ & $\begin{array}{l}\text { Proportion of patients } \\
\text { experiencing improvement of } \geq 1 \\
\text { point from baseline to final visit: } \\
32 \%\end{array}$ \\
\hline
\end{tabular}

Data in bold face are p values. btwn: between; PO: orally; qd: every day; qw: every week; SQ: subcutaneous; BSA: body surface area; N: number; NAPSI: Nail Psoriasis Severity Index; NR: not reported; PUVA: psoralen + ultraviolet A; CsA: cyclosporin A; MTX: methotrexate; ADM: adalimumab; IFX: infliximab; NB-UVB: narrow band ultraviolet B; PASI: Psoriasis Area and Severity Index; PGA: physician global assessment.

mab $1,9,14,22,23,24,30,31,32,33,34,35$ were highly efficacious in treating psoriatic nail disease. Larger studies are necessary to determine comparative effectiveness of these agents $9,14,22,23,24$.
Ustekinumab, an anti-IL-12/23 monoclonal antibody, was highly effective in treating nail psoriasis, when weight-based dosing was used $36,37,38,39,40$. Limited data 
Table 1D. Biologic therapies for nail psoriasis.

\begin{tabular}{|c|c|c|c|c|c|}
\hline Study & Therapy & $\begin{array}{l}\text { Study Type } \\
\text { and Population }\end{array}$ & Outcome Measure & Baseline & Results \\
\hline $\begin{array}{l}\text { Demirsoy } 2013^{14} \text {, } \\
\text { Turkey }\end{array}$ & $\begin{array}{l}\text { IFX, ADM, or ETN vs } \\
\text { MTX, vs narrow-band } \\
\text { UVB (NB-UVB), vs } \\
\text { acitretin, vs no treatment }\end{array}$ & Comparative, $\mathrm{N}=87$ & NAPSI at wks 0,16 & $\begin{array}{l}\text { Any type of skin psoriasis } \\
\text { with nail involvement }\end{array}$ & $\begin{array}{l}\text { Mean NAPSI score at wk 0: } \\
\text { Biologics: } 36.5, \text { MTX: } 25.1 \text {, } \\
\text { NB-UVB: } 22.5 \text {, Acitretin: } \\
\text { 23.8, Control: } 21.3 \text {; wk 16: } \\
\text { Biologics: } 7.9, \mathbf{p}=\text { significant } \\
\text { c/w control but specific value } \\
\text { NR, MTX: } 20.5, \mathbf{N S} \mathbf{c} / \mathbf{w} \\
\text { control, NB-UVB: } 17, \mathbf{N S ~ c / w} \\
\text { control, Acitretin: } 17.9, \mathbf{N S} \mathbf{c} / \mathbf{w} \\
\text { control; Control: } 18.3\end{array}$ \\
\hline $\begin{array}{l}\text { Sola-Ortigosa } \\
2012^{17}, \text { Spain }\end{array}$ & $\begin{array}{l}\text { ADM (initial } 80-\mathrm{mg} \text { dose } \\
\text { SQ at wk } 0 \rightarrow 40 \mathrm{mg} \text { SQ at } \\
\text { wk 1, then eow) }\end{array}$ & Retrospective, $N=15$ & NAPSI at wks 0,24 & $\begin{array}{l}\text { Moderate-to-severe } \\
\text { plaque psoriasis, } \\
\text { failed to respond to } \\
\text { conventional systemic } \\
\text { treatments or other } \\
\text { biological agents, } \\
\text { in which ADM therapy } \\
\text { was indicated }\end{array}$ & $\begin{array}{l}\text { Mean NAPSI score at baseline } \\
\text { vs wk } 24: 18.9 \pm 12.2 \text { vs } 8.2 \pm \\
4.7, \mathbf{p}=\mathbf{0 . 0 0 1}\end{array}$ \\
\hline $\begin{array}{l}\text { Leonardi } 2011^{18} \text {, } \\
\text { USA, Canada }\end{array}$ & $\begin{array}{l}\text { ADM (initial } 80-\mathrm{mg} \text { dose } \\
\text { SQ at wk } 0 \rightarrow 40 \mathrm{mg} \text { SQ } \\
\text { eow wks } 1-15 ; \rightarrow \text { Pbo at } \\
\text { wk } 16 \rightarrow 40 \mathrm{mg} \mathrm{SQ} \text { eow } \\
\text { wks } 17-27 \text { ), vs Pbo } \\
\text { (crossover to ADM } 80 \mathrm{mg} \\
\text { SQ at wk } 16 \rightarrow 40 \mathrm{mg} \mathrm{SQ} \\
\text { eow, wks } 17-27 \text { ) }\end{array}$ & $\begin{array}{l}\text { Randomized, Pbo- } \\
\text { controlled, double-blind } \\
\text { (16 wks); open-label } \\
\text { 12-wk extension, } \mathrm{N}=72\end{array}$ & $\begin{array}{l}\text { NAPSI at wks } \\
0,8,16,28\end{array}$ & $\begin{array}{l}\text { Chronic plaque } \\
\text { psoriasis on hands } \\
\text { and/or feet with } \\
\text { PGA of hands/feet } \\
\text { of at least "moderate" } \\
\text { severity }\end{array}$ & $\begin{array}{l}\text { Mean NAPSI score (target nail): } \\
\text { Baseline: ADM: } 3.9 \pm 2.0 ; \\
\text { Pbo: } 3.3 \pm 1.8 \text {; Mean } \% \\
\text { NAPSI improvement: Wk 16: } \\
\text { ADM: } 50 \%, \text { Pbo: } 8 \% \text {, } \\
\text { p = 0.02 btwn groups; } \\
\text { Wk 28: ADM: } 54 \% \text {, } \\
\text { Pbo (switched to ADM at } \\
\text { wk 16): } 38 \%\end{array}$ \\
\hline $\begin{array}{l}\text { Rigopoulos } 2010^{19} \text {, } \\
\text { Greece }\end{array}$ & $\begin{array}{l}\text { ADM (initial } 80-\mathrm{mg} \text { dose SQ } \\
\text { at wk } 0 \rightarrow 40 \mathrm{mg} \text { SQ at wk } \\
1 \rightarrow 40 \mathrm{mg} \text { SQ q2wks) }\end{array}$ & Open-label, $\mathrm{N}=21$ & $\begin{array}{l}\text { Mean NAPSI } \\
\text { at wks } 0,12 \text {, and } 24\end{array}$ & $\begin{array}{l}\text { Severe plaque psoriasis } \\
\text { with nail involvement }\end{array}$ & $\begin{array}{l}\text { Mean NAPSI score at baseline: } \\
\text { Psoriasis patients: Fingernails: } \\
10.57 \pm 1.21 \text {; Toenails: } 14.57 \pm \\
\text { 2.50, PsA patients: Fingernails: } \\
23.86 \pm 2.00 \text {; Toenails: } 29.29 \pm \\
\text { 2.87, Mean NAPSI score at wk } \\
\text { 24: Psoriasis patients: } \\
\text { Fingernails: } 1.57 \pm 0.20 \text {; } \\
\text { Toenails: } 4.14 \pm 1.58, \text { PsA } \\
\text { patients: Fingernails: } 3.23 \pm \\
0.32 \text {; Toenails: } 10.00 \pm 1.40\end{array}$ \\
\hline $\begin{array}{l}\text { Van den Bosch } \\
2010^{20} \text {, Belgium, } \\
\text { Germany, France, } \\
\text { UK, Norway, } \\
\text { Denmark, Sweden, } \\
\text { Finland, Ireland }\end{array}$ & $\begin{array}{l}\text { ADM ( } 40 \mathrm{mg} \mathrm{SQ} \\
\text { eow } \times 12 \mathrm{wks})\end{array}$ & Open-label, $\mathrm{N}=442$ & $\begin{array}{l}\text { NAPSI at wks } \\
0,12,20\end{array}$ & $\begin{array}{l}\text { Diagnosis of PsA, } \\
\text { previous treatment with } \\
>1 \text { DMARD }\end{array}$ & $\begin{array}{l}\text { Improvement }>50 \% \text { in } \\
\text { NAPSI score at wk } 12 \\
\text { (in patients with baseline } \\
\text { NAPSI > 10): } 54.2 \% \text {, } \\
\text { Median NAPSI: Wk 12: } \\
\text { 5, Wk 20: } 1\end{array}$ \\
\hline $\begin{array}{l}\text { Rudwaleit } 2010^{21} \text {, } \\
\text { Germany }\end{array}$ & $\begin{array}{l}\text { ADM ( } 40 \text { mg SQ eow } \\
\times 12 \text { wks) }\end{array}$ & $\begin{array}{l}\text { Open-label, N = } 442 \\
\text { (with PsA) }\end{array}$ & NAPSI at wks 0,12 & $\begin{array}{l}\text { History of anti-TNF } \\
\text { treatment [IFX, ETN, } \\
\text { or both] and failure of } \\
1 \text { or more DMARD } \\
\text { for PsA }\end{array}$ & $\begin{array}{l}\text { Median change in NAPSI score } \\
\text { at wk 12: No prior ETN/IFX: } \\
-6 \text { (range }-14 \text { to }-2 \text { ), Prior } \\
\text { ETN/IFX: }-6 \text { (range }-15 \text { to }-1 \text { ), } \\
\text { NS btwn groups }\end{array}$ \\
\hline $\begin{array}{l}\text { Ozmen } 2013^{22} \text {, } \\
\text { Turkey }\end{array}$ & $\begin{array}{l}\text { ADM (initial } 80 \text {-mg dose } \\
\text { SQ at wk } 0 \rightarrow 40 \mathrm{mg} \text { SQ } \\
\text { eow starting wk } 1 \text { ), vs ETN } \\
(50 \mathrm{mg} \text { SQ biw } \times 12 \text { wks } \rightarrow \\
50 \mathrm{mg} \text { SQ qw), vs IFX ( } 5 \mathrm{mg} / \mathrm{kg} \\
\text { IV at wks } 0,2,6 \text {, then } \mathrm{q} 8 \mathrm{wks} \\
\text { to wk } 46)\end{array}$ & $\begin{array}{l}\text { Randomized, } \\
\text { open-label, } \mathrm{N}=28\end{array}$ & $\begin{array}{l}\text { NAPSI at wks } \\
0,12,24,36,48\end{array}$ & $\begin{array}{l}\text { Moderate-to-severe } \\
\text { nail psoriasis, failed } \\
\text { other systemic therapies }\end{array}$ & $\begin{array}{l}\text { Mean improvement in NAPSI } \\
\text { score at week 48: ADM: } 53.8 \% \text {, } \\
\text { ETN: } 57.3 \%, \text { IFX: } 40.4 \% \text {, } \\
\text { CI not reported; authors } \\
\text { report difference NS }\end{array}$ \\
\hline
\end{tabular}


Table 1D. Continued

\begin{tabular}{|c|c|c|c|c|c|}
\hline Study & Therapy & $\begin{array}{l}\text { Study Type } \\
\text { and Population }\end{array}$ & Outcome Measure & Baseline & Results \\
\hline $\begin{array}{l}\text { Saraceno } 2013^{23} \text {, } \\
\text { Italy }\end{array}$ & $\begin{array}{l}\text { ADM (initial } 80 \text {-mg dose } \\
\text { SQ at wk } 0 \rightarrow 40 \mathrm{mg} \mathrm{SQ} \\
\text { eow wks } 1-24), \text { vs ETN } \\
(50 \mathrm{mg} \text { SQ biw } \times 12 \text { wks } \\
\rightarrow 25 \mathrm{mg} \text { SQ biw } \times 12 \text { wks), } \\
\text { vs IFX ( } 5 \mathrm{mg} / \mathrm{kg} \mathrm{IV} \mathrm{at} \mathrm{wks} \\
0,2,6 \text {, then q8wks to wk } 24)\end{array}$ & Open-label, $\mathrm{N}=60$ & NAPSI at wks $0,14,24$ & $\begin{array}{l}\text { Moderate-to-severe } \\
\text { plaque psoriasis } \\
\text { or PsA, failed } \geq 2 \\
\text { systemic conventional } \\
\text { treatments, NAPSI } \\
\text { score }>15\end{array}$ & $\begin{array}{l}\text { Mean NAPSI score at baseline } \\
\text { vs wk } 14 \text { : ADM: } 33.1 \pm 14.9 \\
\text { vs } 21.0 \pm 8.91, \mathbf{p} \leq \mathbf{0 . 0 1}, \text { ETN: } \\
34.8 \pm 12.38 \text { vs } 23.6 \pm 10.43, \\
\mathbf{p} \leq \mathbf{0 . 0 1} \text { IFX: } 33.3 \pm 9.76 \\
\text { vs } 14.9 \pm 4.20, \mathbf{p} \leq \mathbf{0 . 0 1} \text {; Mean } \\
\text { NAPSI score at wk } 14 \text { vs wk } \\
24 \text { : ADM: } 21.0 \pm 8.91 \text { vs } 11.4 \pm \\
4.6, \mathbf{p} \leq \mathbf{0 . 0 0 0 2 , ~ E T N : ~} 23.6 \pm \\
10.43 \text { vs } 10.6 \pm 5.25, \mathbf{p} \leq \\
\mathbf{0 . 0 0 1 6}, \text { IFX: } 14.9 \pm 4.20 \text { vs } 3.1 \\
\pm 3.27, \mathbf{p} \leq \mathbf{0 . 0 0 0 0 1} \text {; At week } \\
14, \text { IFX had better efficacy than } \\
\text { ADM and ETN, } \mathbf{p}<\mathbf{0 . 0 5}\end{array}$ \\
\hline $\begin{array}{l}\text { Kyriakou } 2013^{24} \text {, } \\
\text { Greece }\end{array}$ & $\begin{array}{l}\text { ADM (initial } 80-\mathrm{mg} \text { dose } \\
\text { SQ at wk } 0 \rightarrow 40 \mathrm{mg} \mathrm{SQ} \text { at } \\
\text { wk } 1 \rightarrow 40 \mathrm{mg} \text { SQ q2 } 2 \mathrm{ks} \\
\text { thereafter), vs ETN ( } 50 \mathrm{mg} \\
\text { SQ biw } \times 12 \mathrm{wks} \rightarrow 50 \mathrm{mg} \\
\text { SQ qw), vs IFX }(5 \mathrm{mg} / \mathrm{kg} \\
\text { IV at wks } 0,2,8 \text { then q8wks }\end{array}$ & $\begin{array}{l}\text { Open-label, } \\
\text { retrospective, } N=12\end{array}$ & $\begin{array}{l}\text { NAPSI at wks } \\
0,12,24,48\end{array}$ & $\begin{array}{l}\text { Moderate-to-severe } \\
\text { plaque psoriasis, } \\
\text { PASI }>10, \text { NAPSI }>10\end{array}$ & $\begin{array}{l}\text { Mean NAPSI score at baseline } \\
\text { vs wk } 48: \text { IFX: } 80.50 \pm 45.19 \text { vs } \\
4.58 \pm 3.67, \mathbf{p}=\mathbf{0 . 0 0 2}, \mathrm{ADM} \text { : } \\
82.64 \pm 42.35 \text { vs } 9.57 \pm 4.51, \\
\mathbf{p}=\mathbf{0 . 0 0 1}, \text { ETN: } 82.76 \pm 48.06 \\
\text { vs } 6.61 \pm 4.29, \mathbf{p}=\mathbf{0 . 0 0 1}\end{array}$ \\
\hline
\end{tabular}

Mease $2014^{25}$, North America, Latin America, Western Europe, Central/Eastern Europe

Ortonne $2013^{26}$ Austria, France, Greece, Italy

Luger 200927, Germany, UK, Belgium
CZP (200 mg SQ q2wks)

vs CZP (400 mg SQ q4wks) vs $\mathrm{Pbo}(0.9 \%$ saline $)$
ETN (50 mg SQ biw $\times 12$ wks $\rightarrow 50 \mathrm{mg} \mathrm{qw} \times 12 \mathrm{wks}$ [biw/qw]), vs ETN (50 mg $\mathrm{SQ}$ qw $\times 24 \mathrm{wks}[\mathrm{qw} / \mathrm{qw}])$

ETN (25 mg SQ biw $\times 54$ wks), vs Interrupted ETN (initial 50-mg dose SQ biw $\times 12$ wks max or until PGA $\leq 2$; if relapse $(\mathrm{PGA} \geq 3)$, $25 \mathrm{mg}$ ETN SQ biw until response)

Kavanaugh 200928, Golimumab (GLB, 50 mg SQ USA, Canada, Belgium, Poland, Spain, UK q4wks $\times 20$ wks), vs GLB (100 mg SQ q4wks ×20 wks), vs Pbo
Randomized, doubleblind, Pbo-controlled to week 24 , doseblind to week 48 , open-label to week 216, $\mathrm{N}=409(73.3 \%$ with nail disease at baseline)

Randomized, open-label, NAPSI at wks 0, 12, 24 $\mathrm{N}=72$

Randomized, open-label, $\mathrm{N}=771$ (564 with nail psoriasis)

Randomized, doubleblind, Pbo-controlled phase $3, \mathrm{~N}=405$

Modified NAPSI (target fingernail) at wks 0,24

NAPSI at wks time of discontinuation

NAPSI at wks $0,14,24$ $0,12,24,56$, or at Same as above

Randomized, doubleblind, Pbo-controlled phase $3, \mathrm{~N}=405$
Patients with adultonset PsA of at least 6 months' duration, active joint disease, failed $\geq 1$ DMARD, of psoriasis, nail disease at baseline Moderate-to-severe plaque psoriasis, previously failed 1 form of systemic therapy for nail psoriasis Psoriasis with $\mathrm{BSA} \geq$ $10 \%$, PGA $\geq 3$, previously failed usual care documented history

Mean NAPSI score at baseline: CZP $200 \mathrm{mg}: 3.1 \pm 1.8$, CZP 400 mg: $3.4 \pm 2.2$, Pbo: $3.4 \pm$ 2.2; Modified NAPSI score change from baseline at wk 24 : CZP 200 mg: $-1.6, \mathbf{p}=\mathbf{0 . 0 0 3}$ c/w pbo, CZP $400 \mathrm{mg}:-2.0$, p $<0.001$ c/w pbo, Pbo: -1.1 Mean improvement in NAPSI score (target nail) at baseline vs wk 24: biw/qw: 6.0 vs 1.7 ,

$\mathbf{p}<\mathbf{0 . 0 0 0 1}$, qw/qw: 5.8 vs 1.4 , $\mathrm{p}<0.0001$

Mean NAPSI score at baseline vs wk 12 (pooled continuous and interrupted therapy): Patients with nail psoriasis: 4.64 vs $3.30, \mathbf{p}<\mathbf{0 . 0 0 0 1}$

Mean NAPSI score (target nail) at baseline: GLB $50 \mathrm{mg}$ : $4.7 \pm 2.2$, GLB $100 \mathrm{mg}$ : $4.6 \pm 2.1$, Pbo: $4.4 \pm 2.2$; Median \% change in NAPSI: Wk 14: GLB $50 \mathrm{mg}$ : 25\%, GLB $100 \mathrm{mg}$ : 43\%, Pbo: 0\%; Wk 24: GLB $50 \mathrm{mg}$ : 33\%; GLB 100 mg: 54\%; Pbo: $0 \%$

NAPSI at wk 0, $52 \quad$ Patients negative for rheumatoid factor, had active PsA and plaque psoriasis despite therapy with DMARD or NSAID, no previous treatment with TNF antagonists, rituximab, natalizumab, or cytotoxic agents
Mean NAPSI score (target nail) at baseline: GLB $50 \mathrm{mg}$ : $4.7 \pm$ 2.2, GLB $100 \mathrm{mg}: 4.6 \pm 2.1$, Pbo: $4.4 \pm 2.2$; Mean $\%$ change in NAPSI score at wk 52: GLB $50 \mathrm{mg}: 51.6 \pm 46.8$, GLB 100 mg: $65.8 \pm 51.9$, GLB pooled: $59.2 \pm 50.0$, Pbo: $56.2 \pm 48.1$ 


\begin{tabular}{|c|c|c|c|c|c|}
\hline Study & Therapy & $\begin{array}{l}\text { Study Type } \\
\text { and Population }\end{array}$ & Outcome Measure & Baseline & Results \\
\hline $\begin{array}{l}\text { Fabroni } 2011^{30} \text {, } \\
\text { Italy }\end{array}$ & $\begin{array}{l}\text { IFX }(5 \mathrm{mg} / \mathrm{kg} \text { IV at wks } \\
0,2,6, \text { then q8wks to wk } 38)\end{array}$ & $\begin{array}{l}\text { Open-label, uncontrolled } \\
\text { retrospective study without } \\
\text { comparison group, } \mathrm{N}=121 \\
\text { (61 with nail psoriasis) }\end{array}$ & $\begin{array}{l}\text { NAPSI at wks } \\
0,14,28,38\end{array}$ & $\begin{array}{l}\text { Moderate-to-severe } \\
\text { psoriasis (PASI } \geq 10 \text { ) } \\
\text { or PsA for } \geq 1 \text { year with } \\
\text { nail involvement, } \\
\text { previously failed } \geq 2 \\
\text { traditional systemic therapie }\end{array}$ & $\begin{array}{l}\text { Mean NAPSI score at baseline: } \\
49.7 \pm 26.0 \text {; Mean NAPSI score } \\
\text { at wk 14: } 18.6 \pm 9.4 \text {, wk } 22 \text { : } \\
9.5 \pm 4.7 \text {, wk } 38: 7.2 \pm 4.9 \\
\text { es }\end{array}$ \\
\hline Torii $2010^{33}$, Japan & $\begin{array}{l}\text { IFX }(5 \mathrm{mg} / \mathrm{kg} \text { IV at wks } \\
0,2,6,14 \text { then q8wks to } \\
\text { wk } 62) \text {, vs Pbo [crossover at } \\
\text { wk } 16 \text { with IFX ( } 5 \mathrm{mg} / \mathrm{kg}) \\
\text { IV at wks } 18,22 \text {, then q8wks } \\
\text { to wk } 62 \text { ] }\end{array}$ & $\begin{array}{l}\text { Randomized, double-blind, } \\
\text { pbo-controlled, phase } 3 \text {, } \\
\mathrm{N}=54\end{array}$ & $\begin{array}{l}\text { NAPSI at wks } \\
0,10,14,26,42,66\end{array}$ & $\begin{array}{l}\text { Moderate-to-severe plaque } \\
\text { psoriasis } \geq 6 \text { months, } \\
\text { PASI } \geq 12, \text { BSA } \geq 10 \%\end{array}$ & $\begin{array}{l}\text { Mean change in NAPSI score } \\
\text { (change from baseline) at wk } \\
\text { 10: IFX: } 1.4 \pm 2.2, \text { Pbo: }-0.3 \pm \\
\text { 1.0; wk 14: IFX: } 1.6 \pm 2.0, \text { Pbo: } \\
-0.6 \pm 0.8 \text {; wk } 26: \text { IFX: } 2.2 \pm \\
2.3 \text {, Pbo } \rightarrow \text { IFX: } 0.7 \pm 1.3 \text {; wk } 42 \text { : } \\
\text { IFX: } 2.1 \pm 2.0, \text { Pbo } \rightarrow \text { IFX: } 1.9 \pm \\
0.6 \text {; wk } 66: \text { IFX: } 2.6 \pm 2.0, \text { Pbo } \\
\rightarrow \text { IFX: } 2.4 \pm 1.0\end{array}$ \\
\hline $\begin{array}{l}\text { Rich } 2008^{34} \text {, } \\
\text { USA; Germany; UK }\end{array}$ & $\begin{array}{l}\text { IFX }(5 \mathrm{mg} / \mathrm{kg} \mathrm{IV} \mathrm{at} \mathrm{wks} \\
0,2 \text { and } 6 \rightarrow 5 \mathrm{mg} / \mathrm{kg} \text { IV } \\
\text { q8wks to wk } 46), \text { vs Pbo } \\
\text { (crossover to IFX at } \\
\text { wks } 24,26,30,38 \text {, and } 46 \text { ) }\end{array}$ & $\begin{array}{l}\text { Randomized, double-blind, } \\
\text { pbo-controlled, phase } 3 \text {, } \\
\mathrm{N}=373 \text { ( } 305 \text { with nail } \\
\text { psoriasis) }\end{array}$ & $\begin{array}{l}\text { NAPSI at wks } \\
0,10,24\end{array}$ & $\begin{array}{l}\text { Psoriasis for } \geq 6 \\
\text { months, PASI } \geq 12, \\
\text { BSA } \geq 10 \% \text { with nail } \\
\text { involvement }\end{array}$ & $\begin{array}{l}\text { Mean NAPSI score at baseline: } \\
\text { IFX: } 4.6 \pm 2.0, \text { Pbo: } 4.3 \pm 1.9 \text {; } \\
\text { Mean } \% \text { improvement in } \\
\text { NAPSI score: wk 10: IFX: } \\
\text { 26.8\%, Pbo: }-7.7 \%, \mathbf{p}<\mathbf{0 . 0 0 1} \\
\text { btwn groups; wk } 24 \text { : IFX: } \\
\text { 57.2\%, Pbo: }-4.1 \%, \mathbf{p}<\mathbf{0 . 0 0 1} \\
\text { btwn groups }\end{array}$ \\
\hline $\begin{array}{l}\text { Patsatsi } 2013^{36} \text {, } \\
\text { Greece }\end{array}$ & $\begin{array}{l}\text { UST ( } 45 \mathrm{mg} \text { at wks } 0,4 \\
\text { and then q12 weeks } \\
\text { thereafter; } 90 \mathrm{mg} \text { in patients } \\
\text { with body weight }>100 \mathrm{~kg} \text { ) }\end{array}$ & $\begin{array}{l}\text { Nonrandomized, open- } \\
\text { label, uncontrolled, } \\
\mathrm{N}=27\end{array}$ & $\begin{array}{l}\text { NAPSI at wks } \\
0,16,28,40\end{array}$ & $\begin{array}{l}\text { Moderate-to-severe } \\
\text { psoriasis (PASI } \geq 10 \text { ) } \\
\text { with nail involvement }\end{array}$ & 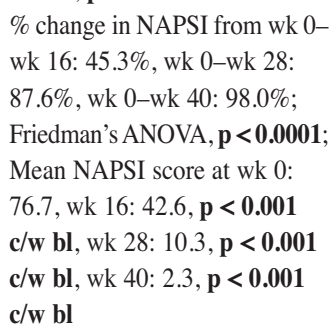 \\
\hline $\begin{array}{l}\text { Rich } 2014^{37} \text {, } \\
\text { USA, Canada, } \\
\text { Netherlands, } \\
\text { Belgium }\end{array}$ & $\begin{array}{l}\text { UST ( } 45 \mathrm{mg} \text { SQ at } \\
\text { wks } 0,4,16,28) \text {, } \\
\text { vs UST }(90 \mathrm{mg} \text { SQ } \\
\text { at wks } 0,4,16,28) \text {, } \\
\text { vs Pbo (crossover to UST } \\
45 \mathrm{mg} \text { or } 90 \mathrm{mg} \text { at wks } \\
12,16,28) \text {. At wk } 40, \\
\text { those with PASI75 } \\
\text { re-randomized to } \\
\text { continue maintenance } \\
\text { dosing or receive Pbo }\end{array}$ & $\begin{array}{l}\text { Randomized, double- } \\
\text { blinded, Pbo-controlled, } \\
\text { phase } 3, \mathrm{~N}=766 \\
\text { ( } 545 \text { with nail psoriasis) }\end{array}$ & $\begin{array}{l}\text { NAPSI at wks } \\
0,12,24\end{array}$ & $\begin{array}{l}\text { Moderate-to- } \\
\text { severe psoriasis }\end{array}$ & $\begin{array}{l}\text { Mean } \% \text { improvement in } \\
\text { NAPSI score at wk } 12 \text { : } \\
\text { UST } 45 \mathrm{mg}: 26.7 \% \text {, } \\
\text { p < 0.001 c/w pbo, UST } \\
90 \mathrm{mg}: 24.9 \%, \mathbf{p}=\mathbf{0 . 0 0 1} \\
\text { c/w pbo; wk } 24: \text { UST } 45 \mathrm{mg}: \\
46.5 \% \text {; UST } 90 \mathrm{mg}: 48.7 \%\end{array}$ \\
\hline
\end{tabular}




\begin{tabular}{|c|c|c|c|c|c|}
\hline Study & Therapy & $\begin{array}{l}\text { Study Type } \\
\text { and Population }\end{array}$ & Outcome Measure & Baseline & Results \\
\hline $\begin{array}{l}\text { Vitiello } 2013^{38} \text {, } \\
\text { USA }\end{array}$ & $\begin{array}{l}\text { UST }(45 \mathrm{mg} \text { at wks } \\
0,4 \text { and then q12 } \\
\text { weeks thereafter; } \\
90 \mathrm{mg} \text { used in } 5 \\
\text { patients with body } \\
\text { weight }>100 \mathrm{~kg} \text { ) }\end{array}$ & Case series, $\mathrm{N}=13$ & $\begin{array}{l}\text { NAPSI at wks } \\
0,4,12\end{array}$ & $\begin{array}{l}\text { PsA for an average } \\
16 \text { years, failed } \geq 4 \\
\text { biologics }\end{array}$ & $\begin{array}{l}\text { Mean NAPSI score at wk 0: } \\
22.3 \text {, wk 4: } 19.5 \text {, wk } 12 \text { : } \\
\text { 14.8; Mean percentage } \\
\text { reduction in NAPSI from } \\
\text { baseline to wk 14: } 31.8 \%\end{array}$ \\
\hline $\begin{array}{l}\text { Igarashi } 2012^{39} \text {, } \\
\text { Japan }\end{array}$ & $\begin{array}{l}\text { UST ( } 45 \mathrm{mg} \text { SQ at } \\
\text { wks } 0,4 \text {, then q12wks } \\
\text { to wk 52), vs UST } \\
\text { ( } 90 \mathrm{mg} \text { SQ at wks } \\
0,4 \text {, then q12wks to } \\
\text { wk } 52 \text { ), vs Pbo } \\
\text { (crossover at wk } 12 \text { to } \\
\text { either UST } 45 \text { mg or } \\
90 \text { mg SQ, wks } 16 \text {, } \\
28,40,52 \text { ) }\end{array}$ & $\begin{array}{l}\text { Randomized, double- } \\
\text { blind, Pbo-controlled, } \\
\text { phase } 2 / 3, \mathrm{~N}=158\end{array}$ & $\begin{array}{l}\text { NAPSI at wks } \\
0,12,64\end{array}$ & $\begin{array}{l}\text { Moderate-to-severe } \\
\text { plaque psoriasis } \geq \\
6 \text { months, PASI } \geq \\
12, \text { BSA } \geq 10 \%\end{array}$ & $\begin{array}{l}\text { Mean NAPSI score at } \\
\text { baseline (if nail psoriasis } \\
\text { present): UST } 45 \mathrm{mg} \text { : } \\
3.7 \pm 1.8 \text {, UST } 90 \mathrm{mg} \text { : } \\
4.1 \pm 2.0 \text {, Pbo: } 4.6 \pm 2.5 \\
\text { Mean } \% \text { improvement in } \\
\text { NAPSI score at wk } 64 \text { : } \\
\text { UST } 45 \mathrm{mg}: 56.6 \pm 43.2 \% \text {, } \\
\text { UST } 90 \mathrm{mg}: 67.8 \pm 37.5 \%\end{array}$ \\
\hline $\begin{array}{l}\text { Rigopoulos } 2011^{40} \text {, } \\
\text { Greece }\end{array}$ & $\begin{array}{l}\text { UST }(45 \mathrm{mg} \text { SQ if } \\
<100 \mathrm{~kg} \text { or } 90 \mathrm{mg} \mathrm{SQ} \\
\text { if }>100 \mathrm{~kg} \text { at wks } 0, \\
4,16 \text { and } 28)\end{array}$ & Open-label, $\mathrm{N}=27$ & $\begin{array}{l}\text { NAPSI at wks } \\
0,4,16,28,40\end{array}$ & $\begin{array}{l}\text { Plaque psoriasis with } \\
\text { fingernail involvement, } \\
\text { failed a systemic } \\
\text { treatment }\end{array}$ & $\begin{array}{l}\text { Mean NAPSI score at wk } 0: \\
19.59 \pm 7.92 \text { (p value } \mathrm{c} / \mathrm{w} \\
\text { bl), wk } 4: 16.96 \pm 6.99, \mathbf{p}< \\
\mathbf{0 . 0 0 1} \text {, wk } 16: 9.70 \pm 4.47, \\
\mathbf{p}<\mathbf{0 . 0 0 1} \text {, wk } 28: 3.85 \pm 3.03, \\
\mathbf{p}<\mathbf{0 . 0 0 1} \text {, wk } 40: 2.00 \pm \\
2.34, \mathbf{p}<\mathbf{0 . 0 0 1}\end{array}$ \\
\hline $\begin{array}{l}\text { Leonardi } 2012^{41} \text {, } \\
\text { USA, Denmark }\end{array}$ & $\begin{array}{l}\text { IXE SQ (10 mg) vs } \\
\text { IXE ( } 25 \mathrm{mg}) \text { vs IXE } \\
(75 \mathrm{mg}) \text { vs IXE ( } 150 \mathrm{mg}) \\
\text { vs Pbo, all at wks } 0,2, \\
4,8,12,16\end{array}$ & $\begin{array}{l}\text { Randomized, double- } \\
\text { blind, pbo-controlled, } \\
\text { phase } 2, \mathrm{~N}=142\end{array}$ & $\begin{array}{l}\text { NAPSI at wks } \\
0,1,2,4,6,8,12\end{array}$ & $\begin{array}{l}\text { Chronic moderate-to- } \\
\text { severe plaque psoriasis } \\
\text { for } \geq 6 \text { months, } \\
\text { PASI } \geq 12, \text { PGA } \geq 3, \\
\text { BSA } \geq 10 \%\end{array}$ & $\begin{array}{l}\text { Mean NAPSI score at } \\
\text { baseline: IXE } 10 \mathrm{mg}: 41.9 \pm \\
\text { 44.8, IXE } 25 \mathrm{mg}: 34.9 \pm \\
\text { 37.7, IXE } 75 \mathrm{mg}: 45.0 \pm \\
\text { 46.9, IXE } 150 \mathrm{mg}: 46.5 \pm \\
51.7, \text { Pbo: } 35.0 \pm 28.1 ; \\
\text { Mean \% change in NAPSI } \\
\text { score at wk 12: IXE } 10 \mathrm{mg}: \\
\text { 14.3 } \pm \text { 97.8, NS c/w Pbo, } \\
\text { IXE } 25 \text { mg: }-24.0 \pm 32.8, \mathbf{N S} \\
\text { c/w Pbo, IXE } 75 \text { mg: }-57.1 \pm \\
36.7, \mathbf{p}<\mathbf{0 . 0 1} \text { c/w Pbo, IXE } \\
\text { 150 mg: }-49.3 \pm 35.9, \mathbf{p}< \\
\mathbf{0 . 0 5} \text { c/w Pbo, Pbo: } 6.8 \pm 41.1\end{array}$ \\
\hline
\end{tabular}

Data in bold face are p values. btwn: between; PO: orally; qd: every day; qw: every week; SQ: subcutaneous; c/w: compared with; adalimumab (ADM); NAPSI: Nail Psoriasis Area Severity Index; narrow-band UVB (NB-UVB); MTX: methotrexate; Pbo: placebo; IFX: infliximab; ETN: etanercept; DMARD: disease modifying antirheumatic drugs; CZP: certolizumab pegol; PGA: physician global assessment; PASI: Psoriasis Area and Severity Index; BSA: body surface area; IXE: ixekizumab; UST: ustekinumab; eow: every other week; biw: biweekly; TNF: tumor necrosis factor; NR: not reported; bl: baseline.

show that IL-17 blockade with ixekinumab (> $75 \mathrm{mg}$ subcutaneously) also appears to be effective ${ }^{41}$.

\section{Combination Therapies ${ }^{11,42}$ (Table IE)}

Literature on combination therapies for nail psoriasis is limited. In 1 single-blind, within-patient trial of PDL ( $595 \mathrm{~nm}, 1.5 \mathrm{~ms}$ pulse duration) plus topical $0.1 \%$ tazarotene cream compared to topical tazarotene alone, a significantly greater mean decrease in nail matrix modified NAPSI score was observed with PDL-tazarotene compared to tazarotene alone ${ }^{42}$.

In a nonrandomized, unblinded study of ADM plus cyclosporine (CSA) compared to ADM monotherapy and CSA monotherapy, $100 \%$ of patients receiving combination therapy reported $>50 \%$ improvement in mean NAPSI score at week 12 compared to patients receiving either CSA (44\%) or ADM $(56 \%)$ alone $e^{11}$.

In conclusion, nail psoriasis results in significant morbidity and warrants adequate treatment. Topical therapies may be an initial option, but their efficacy is modest. Procedural therapies require more investigation to determine their efficacy. Traditional oral therapies, e.g., MTX or CSA, may be helpful at high doses. The most rigorously studied therapies are biologic agents, with evidence suggesting that TNF- $\alpha$ inhibitors and IL-12/23 inhibitors are highly efficacious in treating nail psoriasis. 
Table 1E. Combination therapies for nail psoriasis.

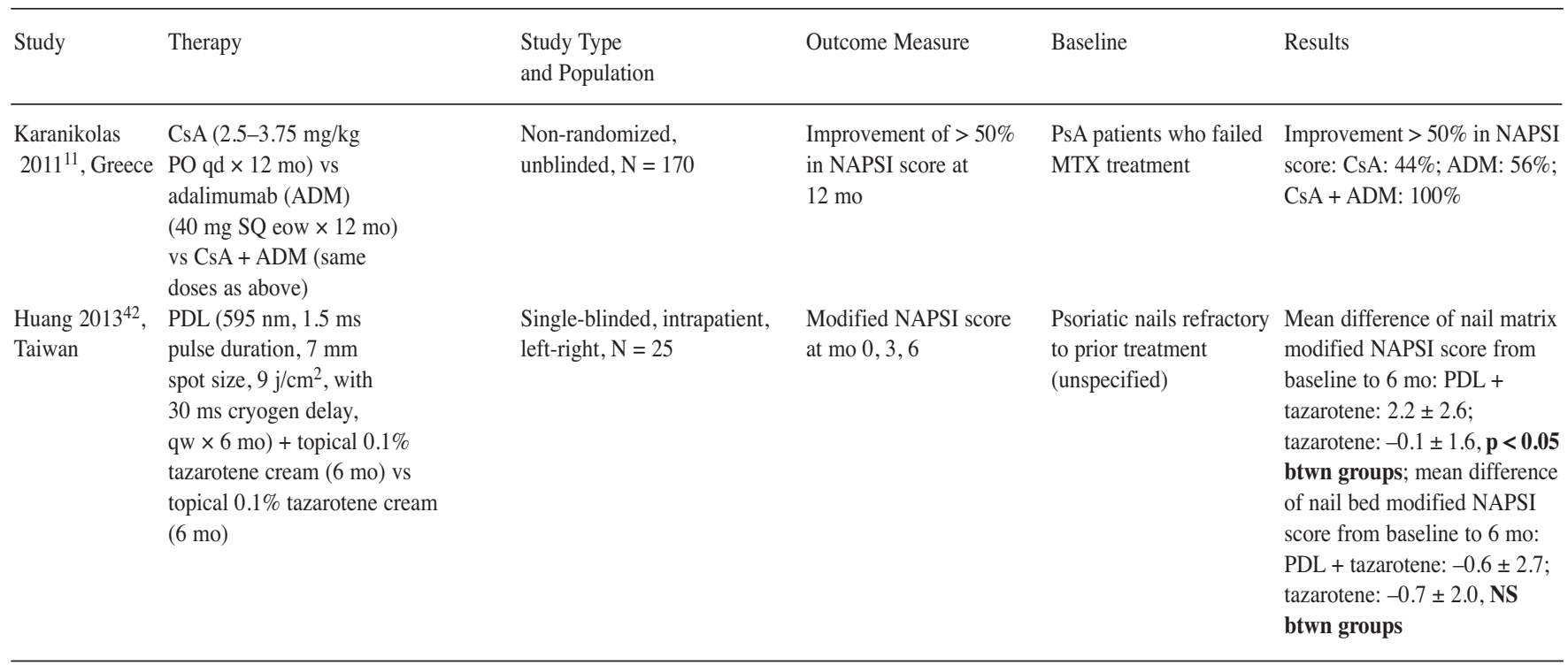

Data in bold face are p values. btwn: between; PO: orally; qd: every day; eow: every other week; qw: every week; SQ: subcutaneous; CsA: cyclosporine; ADM: adalimumab; MTX: methotrexate; NAPSI: Nail Psoriasis Area Severity Index; PDL: pulsed dye laser.

\section{REFERENCES}

1. Cassell S, Kavanaugh AF. Therapies for psoriatic nail disease. A systematic review. J Rheumatol 2006;33:1452-6.

2. Rigopoulos D, Gregoriou S, Daniel CR III, Belyayeva H, Larios G, Verra P, et al. Treatment of nail psoriasis with a two-compound formulation of calcipotriol plus betamethasone dipropionate ointment. Dermatology 2009;218:338-41.

3. Tzung TY, Chen CY, Yang CY, Lo PY, Chen YH. Calcipotriol used as monotherapy or combination therapy with betamethasone dipropionate in the treatment of nail psoriasis. Acta Derm Venereol 2008;88:279-80.

4. De Simone C, Maiorino A, Tassone F, D’Agostino M, Caldarola G. Tacrolimus $0.1 \%$ ointment in nail psoriasis: A randomized controlled open-label study. J Eur Acad Dermatol Venereol 2013;27:1003-6.

5. Fischer-Levancini C, Sanchez-Regana M, Llambi F, Collgros H, Exposito-Serrano V, Umbert-Millet P. Nail psoriasis: Treatment with tazarotene $0.1 \%$ hydrophilic ointment. Actas Dermosifiliogr 2012;103:725-8.

6. Goldust M, Raghifar R. Clinical trial study in the treatment of nail psoriasis with pulsed dye laser. J Cosmet Laser Ther 2013 Oct 16 [Epub ahead of print].

7. Treewittayapoom C, Singvahanont P, Chanprapaph K, Haneke E. The effect of different pulse durations in the treatment of nail psoriasis with 595-nm pulsed dye laser: A randomized, double-blind, intrapatient left-to-right study. J Am Acad Dermatol 2012;66:807-12.

8. Gumusel M, Ozdemir M, Mevlitoglu I, Bodur S. Evaluation of the efficacy of methotrexate and cyclosporine therapies on psoriatic nails: A one-blind, randomized study. J Eur Acad Dermatol Venereol 2011;25:1080-4.

9. Sanchez-Regana M, Sola-Ortigosa J, Alsina-Gibert M, Vidal-Fernandez M, Umbert-Millet P. Nail psoriasis: A retrospective study on the effectiveness of systemic treatments (classical and biological therapy). J Eur Acad Dermatol Venereol 2011;25:579-86.

10. Syuto T, Abe M, Ishibuchi H, Ishikawa O. Successful treatment of psoriatic nails with low-dose cyclosporine administration. Eur J
Dermatol 2007;17:248-9.

11. Karanikolas GN, Koukli EM, Katsalira A, Arida A, Petrou D, Komninou E, et al. Adalimumab or cyclosporine as monotherapy and in combination in severe psoriatic arthritis: Results from a prospective 12-month nonrandomized unblinded clinical trial. J Rheumatol 2011;38:2466-74.

12. Kingsley GH, Kowalczyk A, Taylor H, Ibrahim F, Packham JC, $\mathrm{McHugh} \mathrm{NJ}$, et al. A randomized placebo-controlled trial of methotrexate in psoriatic arthritis. Rheumatology 2012;51:1368-77.

13. Reich K, Langley RG, Papp KA, Ortonne JP, Unnebrink K, Kaul $\mathrm{M}$, et al. A 52-week trial comparing briakinumab with methotrexate in patients with psoriasis. N Engl J Med 2011;365:1586-96.

14. Demirsoy EO, Kiran R, Salman S, Caglayan C, Akturk AS, Bayramgurler D, et al. Effectiveness of systemic treatment agents on psoriatic nails: A comparative study. J Drugs Dermatol 2013;12:1039-43

15. Tosti A, Ricotti C, Romanelli P, Cameli N, Piraccini BM. Evaluation of the efficacy of acitretin therapy for nail psoriasis. Arch Dermatol 2009;145:269-71.

16. Behrens F, Finkenwirth C, Pavelka K, Stolfa J, Sipek-Dolnicar A, Thaci D, et al. Leflunomide in psoriatic arthritis: Results from a large European prospective observational study. Arthritis Care Res 2013;65:464-70.

17. Sola-Ortigosa J, Sanchez-Regana M, Umbert-Millet P. Efficacy of adalimumab in the treatment of psoriasis: A retrospective study of 15 patients in daily practice. J Dermatolog Treat 2012;23:203-7.

18. Leonardi C, Langley RG, Papp K, Tyring SK, Wasel N, Vender R, et al. Adalimumab for treatment of moderate to severe chronic plaque psoriasis of the hands and feet: Efficacy and safety results from REACH, a randomized, placebo-controlled, double-blind trial. Arch Dermatol 2011;147:429-36.

19. Rigopoulos D, Gregoriou S, Lazaridou E, Belyayeva E, Apalla Z, Makris M, et al. Treatment of nail psoriasis with adalimumab: An open label unblinded study. J Eur Acad Dermatol Venereol 2010;24:530-4.

20. Van den Bosch F, Manger B, Goupille P, McHugh N, Rodevand E, Holck $\mathrm{P}$, et al. Effectiveness of adalimumab in treating patients with active psoriatic arthritis and predictors of good clinical responses

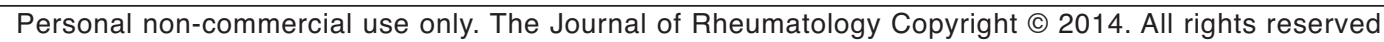


for arthritis, skin and nail lesions. Ann Rheum Dis 2010;69:394-9.

21. Rudwaleit M, Van den Bosch F, Kron M, Kary S, Kupper H.

Effectiveness and safety of adalimumab in patients with ankylosing spondylitis or psoriatic arthritis and history of anti-tumor necrosis factor therapy. Arthritis Res Ther 2010;12:R117.

22. Ozmen I, Erbil AH, Koc E, Tunca M. Treatment of nail psoriasis with tumor necrosis factor-alpha blocker agents: An open-label, unblinded, comparative study. J Dermatol 2013;40:755-6.

23. Saraceno R, Pietroleonardo L, Mazzotta A, Zangrilli A, Bianchi L, Chimenti S. TNF-alpha antagonists and nail psoriasis: An open, 24-week, prospective cohort study in adult patients with psoriasis. Expert Opin Biol Ther 2013;13:469-73.

24. Kyriakou A, Patsatsi A, Sotiriadis D. Anti-TNF agents and nail psoriasis: A single-center, retrospective, comparative study. J Dermatolog Treat 2013;24:162-8.

25. Mease PJ, Fleischmann R, Deodhar AA, Wollenhaupt J, Khraishi $\mathrm{M}$, Kielar D, et al. Effect of certolizumab pegol on signs and symptoms in patients with psoriatic arthritis: 24-week results of a Phase 3 double-blind randomised placebo-controlled study (RAPID-PsA). Ann Rheum Dis 2014;73:48-55.

26. Ortonne JP, Paul C, Berardesca E, Marino V, Gallo G, Brault Y, et al. A 24-week randomized clinical trial investigating the efficacy and safety of two doses of etanercept in nail psoriasis. $\mathrm{Br} \mathrm{J}$ Dermatol 2013;168:1080-7.

27. Luger TA, Barker J, Lambert J, Yang S, Robertson D, Foehl J, et al. Sustained improvement in joint pain and nail symptoms with etanercept therapy in patients with moderate-to-severe psoriasis. J Eur Acad Dermatol Venereol 2009;23:896-904.

28. Kavanaugh A, McInnes I, Mease P, Krueger GG, Gladman D, Gomez-Reino J, et al. Golimumab, a new human tumor necrosis factor alpha antibody, administered every four weeks as a subcutaneous injection in psoriatic arthritis: Twenty-four-week efficacy and safety results of a randomized, placebo-controlled study. Arthritis Rheum 2009;60:976-86.

29. Kavanaugh A, van der Heijde D, McInnes IB, Mease P, Krueger GG, Gladman DD, et al. Golimumab in psoriatic arthritis: One-year clinical efficacy, radiographic, and safety results from a phase III, randomized, placebo-controlled trial. Arthritis Rheum 2012;64:2504-17.

30. Fabroni C, Gori A, Troiano M, Prignano F, Lotti T. Infliximab efficacy in nail psoriasis. A retrospective study in 48 patients. J Eur Acad Dermatol Venereol 2011;25:549-53.

31. Torii H, Nakagawa H, Japanese Infliximab Study I. Long-term study of infliximab in Japanese patients with plaque psoriasis, psoriatic arthritis, pustular psoriasis and psoriatic erythroderma. J Dermatol 2011;38:321-34.
32. Reich K, Ortonne JP, Kerkmann U, Wang Y, Saurat JH, Papp K, et al. Skin and nail responses after 1 year of infliximab therapy in patients with moderate-to-severe psoriasis: A retrospective analysis of the EXPRESS Trial. Dermatology 2010;22:172-8.

33. Torii H, Nakagawa H, Japanese Infliximab Study I. Infliximab monotherapy in Japanese patients with moderate-to-severe plaque psoriasis and psoriatic arthritis. A randomized, double-blind, placebo-controlled multicenter trial. J Dermatol Sci 2010;59:40-9.

34. Rich P, Griffiths CE, Reich K, Nestle FO, Scher RK, Li S, et al. Baseline nail disease in patients with moderate to severe psoriasis and response to treatment with infliximab during 1 year. J Am Acad Dermatol 2008;58:224-31.

35. Rigopoulos D, Gregoriou S, Stratigos A, Larios G, Korfitis C, Papaioannou D, et al. Evaluation of the efficacy and safety of infliximab on psoriatic nails: An unblinded, nonrandomized, open-label study. Br J Dermatol 2008;159:453-6.

36. Patsatsi A, Kyriakou A, Sotiriadis D. Ustekinumab in nail psoriasis: An open-label, uncontrolled, nonrandomized study. J Dermatolog Treat 2013;24:96-100.

37. Rich P, Bourcier M, Sofen H, Fakharzadeh S, Wasfi Y, Wang Y, et al. Ustekinumab improves nail disease in patients with moderate-to-severe psoriasis: Results from PHOENIX 1. Br J Dermatol 2014;170:398-407.

38. Vitiello M, Tosti A, Abuchar A, Zaiac M, Kerdel FA. Ustekinumab for the treatment of nail psoriasis in heavily treated psoriatic patients. Int J Dermatol 2013;52:358-62.

39. Igarashi A, Kato T, Kato M, Song M, Nakagawa H, Japanese Ustekinumab Study G. Efficacy and safety of ustekinumab in Japanese patients with moderate-to-severe plaque-type psoriasis: Long-term results from a phase $2 / 3$ clinical trial. J Dermatol 2012;39:242-52.

40. Rigopoulos D, Gregoriou S, Makris M, Ioannides D. Efficacy of ustekinumab in nail psoriasis and improvement in nail-associated quality of life in a population treated with ustekinumab for cutaneous psoriasis: An open prospective unblinded study. Dermatology 2011;223:325-9.

41. Leonardi C, Matheson R, Zachariae C, Cameron G, Li L, Edson-Heredia E, et al. Anti-interleukin-17 monoclonal antibody ixekizumab in chronic plaque psoriasis. N Engl J Med 2012;366:1190-9.

42. Huang YC, Chou CL, Chiang YY. Efficacy of pulsed dye laser plus topical tazarotene versus topical tazarotene alone in psoriatic nail disease: A single-blind, intrapatient left-to-right controlled study. Lasers Surg Med 2013;45:102-7. 